\title{
COMMUNICABLE DISEASES REPORT, NSW, FOR MAY 2003
}

\section{TRENDS}

Summaries of case notifications through to May 2003 are shown in Figure 2 and Table 4.

\section{BLOOD-BORNE AND SEXUALLY TRANSMISSIBLE INFECTIONS}

Quarterly Report: HIV notifications to end of March 2003

Figure 1 and Table 1 summarise recent trends in human immunodeficiency virus (HIV) disease in NSW.

\section{Background}

HIV causes acquired immunodeficiency syndrome (AIDS). The virus is spread from person to person, mainly through exposure to the sexual fluid or blood from an infected person. In Australia, people at highest risk of infection include men who have sex with men, injecting drug users, and sex workers. Soon after infection, many people will experience a self-limiting influenza-like illness. The infection then remains silent for many years. In most cases, without treatment the virus will eventually destroy the infected person's immune system, allowing the development of other infections (for example, pneumonia or tuberculosis), and cancer (for example, Kaposi's sarcoma). Wasting disease and dementia can also occur. When these secondary illnesses occur, the person is said to have AIDS.
AIDS was first recognised in NSW in the early 1980s, and has mainly affected men who have sex with men. In NSW, surveillance systems were set up and coordinated nationally to monitor the epidemic. National data on HIV disease is gathered from a range of data sources, including notifications from laboratories and doctors of people with HIV or AIDS, special surveys of sexual health clinics, clients of needle and syringe programs, and special studies of risk factors among populations at high risk.

\section{Surveillance}

NSW Health coordinates surveillance of HIV and AIDS by collating notifications of people newly-diagnosed with HIV infection from diagnostic laboratories, and by seeking notifications of people diagnosed with AIDS from doctors. Under the Public Health Act (NSW), laboratories and doctors are required to notify HIV and AIDS cases using a code for the patient's name and their date of birth.

There are limits to HIV surveillance in that:

- it can only identify people who present to their doctors for testing. Because people can be infected and have no symptoms, they may not present for testing until years after their initial infection;

- there is little identification data on the cases, as a name code is used rather than a full name and address, which makes both the follow up of cases and removal of duplicate notifications of cases difficult.

\section{FIGURE 1}

NOTIFICATION OF HIV INFECTION, AIDS, AND AIDS DEATHS BY YEAR, NSW, 1981 TO MARCH 2003

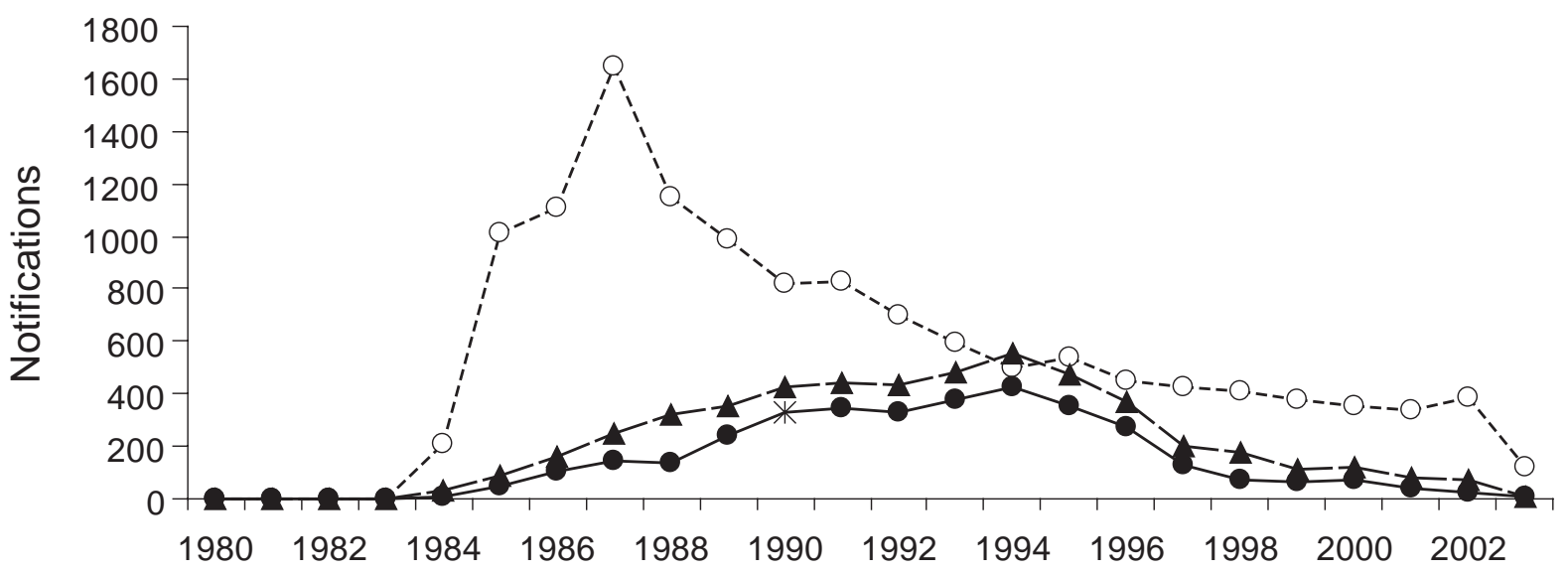

- - - HIV notifications $\rightarrow$ A-AIDS notifications $\rightarrow$ - AIDS deaths

Note: HIV diagnoses are by date of first positive result. 


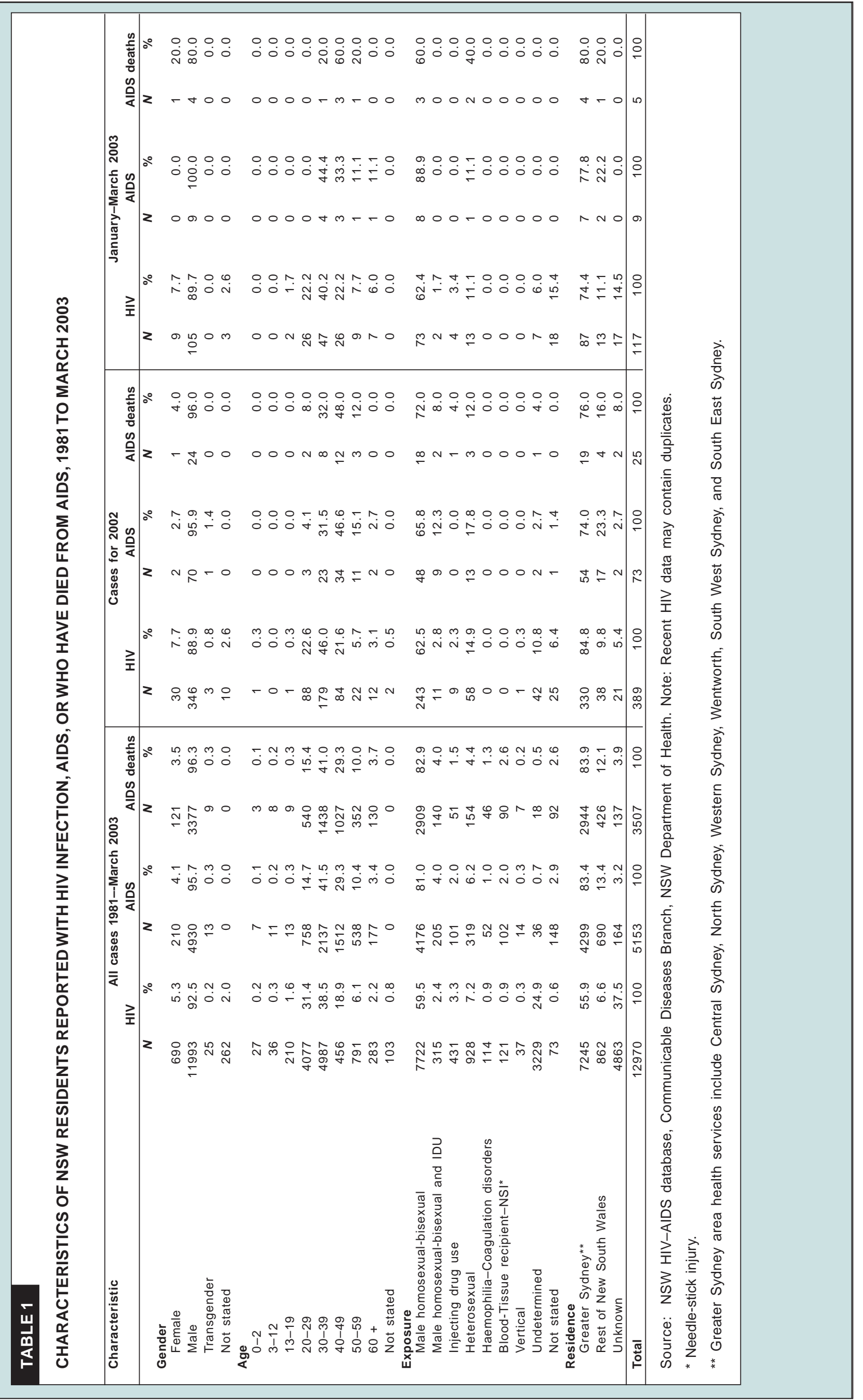


HIV surveillance data should therefore be interpreted with caution, since it measures the number of people who have been newly-diagnosed with HIV infection, rather than new infections of HIV. Changes in the number of new diagnoses may reflect changing patterns of HIV testing or changes in infection rates. Through the process of follow-up of notifications with the doctors of patients, new information about cases comes to light from time to time, which may provide better information about their patient's risk or previous HIV test history. Such new information will result in retrospective changes to the number and risk profiles of the people notified with HIV in NSW.

The number of new HIV diagnoses peaked in the late 1980s and declined in the early 1990s. In recent years the number of new diagnoses has plateaued. For 2001, 341 cases with newly-diagnosed HIV infection were notified in NSW, representing the lowest number to date. For 2002, 389 cases were reported, an increase of 14 per cent on 2001. As previously described, the increase could reflect a real increase in new infections in NSW, or may simply reflect a change in testing patterns of people with HIV. The increase was mainly among people 30-39 years of age and patients who report the risk factors of engaging in male-to-male sex.

In NSW, a number of other indictors suggest that a real increase in the incidence of HIV is likely. These are:

- an increase in reports of gonorrhoea in inner Sydney in recent years (consistent with clinical reports that many of these cases are among men who have sex with men);

- an increase in new syphilis infections in inner Sydney since 2000 (again, many cases are among men who have sex with men).

The number of people notified with AIDS and who have died from AIDS has declined since the mid-1990s, most

\section{GLOSSARY OFTERMS}

New HIV diagnosis refers to a person who is diagnosed for the first time with human immunodeficiency virus (HIV) infection.

Newly acquired HIV infection refers to a person with a new HIV diagnosis who tested HIV negative or reported a seroconversion illness in the 12 months before HIV diagnosis

AIDS refers to a person with HIV infection who develops one of several infections, malignancies, or other medical conditions indicating immune depression consistent with the definition of the acquired immunodeficiency syndrome (AIDS).

AIDS death refers to a person who has died of any cause after being diagnosed with AIDS. likely due to better treatment for HIV infection and the general decline in new HIV infections.

\section{VECTOR-BORNE DISEASES}

Notifications of Ross River and Barmah Forest virus infection increased substantially in the Northern Rivers Area following coastal rains, high tides, and warm weather, all of which are conducive to mosquito breeding.

\section{RESPIRATORY AND OTHER DISEASES}

\section{Meningococcal disease cluster on an aeroplane}

In May, a 68-year-old woman presented to a Central Sydney hospital unwell with headache and fever and a three-week history of respiratory illness. She was diagnosed with meningococcal disease, which was confirmed when a sample of cerebrospinal fluid (CSF) tested positive for Neisseria meningitidis by nucleic acid testing. The public health investigation identified no likely risk for meningococcal disease, but it was noted that the woman had recently flown to Sydney from Los Angeles.

Two days later, an 86-year-old woman presented to a South Western Sydney hospital with shortness of breath. Her condition soon deteriorated. She required ventilation, and she also developed a petechial rash. Gram-negative diplococci (consistent with $N$. meningitidis) were identified in a sample of her CSF. The patient had travelled from Central America to Sydney in the previous week by aeroplane.

Routine follow-up of meningococcal cases includes identification of close contacts, which includes people sitting in adjacent seats to a case on air flights longer than eight hours duration. In following up possible airline contacts for each case, it soon became apparent that both had travelled on the same international flight from Los Angeles to Sydney during their incubation period. The passengers were seated on the same side of the plane, but in different sections; however, the investigation could identify no direct contact between the two either during or off the flight.

A literature search found no reports of transmission of meningococcal disease during air flights. Nonetheless, Australian and United States guidelines recommend that passengers seated adjacent to a meningococcal case on long haul flights should be considered for chemoprophylaxis. ${ }^{1,2}$

An expert teleconference recommended that the public health units provide advice and chemoprophylaxis to those passengers sitting adjacent to, and in rows in front and behind, each passenger, and that a media release be issued alerting other passengers and crew. The airline provided a list of passengers and the Commonwealth Department of Immigration and Multicultural and Indigenous Affairs provided contact details of these passengers from the flight landing cards. 
Further testing showed that both cases were due to N. meningitidis serogroup $\mathrm{B}$, and genotyping results strongly suggest that the two cases were caused by the same serogroup B meningococcus. No further cases related to this airline cluster were subsequently identified.

\section{References}

1. Centers for Disease Control and Prevention. Exposure to patients with meningococcal disease on aircraft, United Sates, 1999-2001. MMWR 2001; 50: 485-489.

2. Communicable Diseases Network Australia. Guidelines for the early clinical and public health management of Meningococcal Disease in Australia. Canberra: Commonwealth Department of Health and Aged Care, 2001.

\section{UPDATE ON SEVERE ACUTE RESPIRATORY SYNDROME INVESTIGATIONS}

An epidemic of atypical pneumonia was first noted in southern China in November 2002. Subsequent transmission to travellers and their contacts led the World Health Organization to issue a global alert on 15 March. The disease, which included fever and respiratory symptoms, was named Severe Acute Respiratory Syndrome (SARS).

The case definition for suspected SARS included a person who visited an affected area or who had close contact with a SARS case in the previous 10 days who develop fever $\left(>38^{\circ} \mathrm{C}\right)$ and cough or difficulty breathing. Probable cases also have chest $\mathrm{x}$-ray changes of pneumonia or Respiratory Distress Syndrome (RDS) in the absence of another cause. However, because no causative pathogen is identified in 40 percent of patients with communityacquired pneumonia in normal circumstances, many patients fitting this definition may well have a disease other than SARS.

A new coronavirus, known as SARS CoV, has now been identified as the pathogen that causes SARS. Specific diagnostic tests for SARS CoV have been developed and are currently being validated. Definitive diagnosis of SARS remains difficult, however, because the tests are not positive in all cases.

Active surveillance for SARS in NSW began on 17 March 2003. Hospitals and general practitioners were alerted to report possible cases to their public health unit and to use SARS infection control precautions. All patients under investigation are reviewed by a national expert committee to determine the probability of SARS in each patient.

As of 13 June, the Communicable Diseases Branch, NSW Department of Health, had been notified of 56 people who have been investigated for possible SARS. Four had onset of fever in February, 15 in March, 20 in April, 14 in May, and three in June. The age range in these 56 patients was from seven months to 81 years. Six (11 per cent) were aged $0-4$ years, six (11 per cent) were aged 5-19 years, 18 (32 per cent) were aged 20-39 years, 18 (32 per cent) were aged $40-59$ years, and eight (14 per cent) were older than 60 years. Thirty-two cases (57 per cent) were males. Thirty-four (61 per cent) resided in metropolitan Sydney and 15 (27 per cent) resided in other parts of NSW. Seven (13 per cent) were international travellers.

All 56 patients reported fever. Fifty patients ( 89 per cent) reported cough. Twenty-five patients (45 per cent) also reported either shortness of breath or difficulty breathing. Forty-eight patients ( 86 per cent) had fever and respiratory symptoms but either had no chest x-ray or their x-ray showed no evidence of pneumonia. SARS is an unlikely diagnosis in this group. An alternative diagnosis has been subsequently confirmed for 13 of these cases: mycoplasma [3], parainfluenza [2], influenza A [1], Epstein-Barr virus [1], rhinovirus [1], E. coli [1], group A streptococcus [1], typhoid [1], and chlamydia pneumoniae [2].

Of the eight patients ( 14 per cent) with pneumonia confirmed by chest x-ray, one has been reported to WHO as a probable SARS case and one is currently in hospital under investigation. Of the remaining six, two had x-ray changes that were inconsistent with SARS, two had alternative diagnoses (influenza A and mycoplasma), one had a limited exposure while in transit, and one had both a limited exposure while in transit and an alternate diagnosis (parainfluenza). All these people have recovered. Thirty-six patients (64 per cent) were hospitalised. None have required intensive care and none have died.

Fifty-five of the 56 patients investigated reported being in an affected country. These countries were: 23 (41 per cent) in Hong Kong, 24 (43 per cent) in Singapore, 12 (21 per cent) in China, three (five per cent) in Taiwan, two (four per cent) in Hanoi, and two (four per cent) in Toronto. Several people had visited more than one affected area. One person's exposure was close contact with another person who was being investigated as a possible SARS case.

The Commonwealth Department of Health and Ageing has introduced SARS Health Information Cards for use at airports for surveillance of inbound flights for possible cases of SARS. These cards are handed to all passengers and air crew entering Australia, who are then required to complete a declaration about their recent travel history, any contact with SARS cases, and any symptoms they have. This enables identification of appropriate people for referral to the registered nurses who remain stationed at Sydney International Airport to review possible SARS cases. In total, the nurses have identified one person who required further medical assessment for SARS. This person had fever and cough and was discharged from hospital the following day.

The Taskforce on SARS (TSARS) continues to meet every two weeks to develop and implement local policies for the surveillance and containment of possible cases of SARS. 


\section{ENTERIC DISEASES}

Viral gastroenteritis occurs frequently in NSW, particularly in winter. Implicated organisms include Norovirus (the virus formally known as Norwalk-like), Rotavirus, and Calicivirus. Viral gastroenteritis symptoms include nausea, vomiting, and diarrhoea. Most symptoms resolve within 48 hours. Infection with viral gastroenteritis can be prevented by thorough hand washing and cleaning of contaminated rooms. Early in May, 34 children attending a school camp were taken to hospital in Macquarie Area Health Service with symptoms consistent with viral gastroenteritis. All the children recovered rapidly. Three other outbreaks of viral gastroenteritis were also reported in May.

South Western Sydney Public Health Unit investigated a large outbreak of Salmonella typhimirium 99 associated with a child's birthday party. Over 60 people became ill after a buffet style meal in a restaurant. The final results of the investigation are pending.

An increase in Salmonella infantis was identified in May. A total of 20 cases were reported, mainly in metropolitan Sydney (seven cases in Central Sydney and six cases in Northern Sydney). Public health unit staff interviewed 12 cases; however, the cause of this outbreak remains unclear.

\section{VACCINE-PREVENTABLE DISEASES}

\section{Pertussis outbreak in a Northern Sydney Boarding School}

In May, the Northern Sydney Public Health Unit (NSPHU) was notified of 31 students at a boarding school with a coughing illness. Most of the 31 boarded at the school, and were in Years 7 and 8. Seven of the students (including six day students and one boarder) had pertussis confirmed by positive IgA serology or throat swab nucleic acid testing. NSPHU recommended pertussis-specific treatment (erythromycin or roxithromycin) and isolation until five days of antibiotics had been taken for any student who had a cough, and also recommended preventive therapy for any dormitory contacts. Letters were provided to the school for all parents, which included information on the symptoms of pertussis and the role of vaccination in preventing future outbreaks of pertussis. School authorities independently elected to close the boarding house and send all 150 boarders from years 7-11 home for five days, as a further control measure. Preventive therapy was recommended to all boarders in Year 7 and 8 to take while at home. No further cases were reported after 30 May, and no spread was identified in the wider community.

\section{Locally acquired measles in South Western Sydney}

In early May, the South Western Sydney Public Health Unit (SWSPHU) was notified by a laboratory of a patient who was positive for measles IgM in a sample taken five days before. (Convalescent serology subsequently demonstrated a rise in measles IgG.) The patient was a 19year-old female who had no evidence of prior measles vaccination. Her illness was consistent with measles. On investigation, SWSPHU found that while infectious the patient had: twice visited a doctor's surgery in South Western Sydney; had visited an accident and emergency department in South Western Sydney; had visited a doctor's surgery in Central Sydney; and had attended school. The patient had no history of recent travel or of overseas visitors.

The SWSPHU and Central Sydney Public Health Unit identified 68 people (including 11 students and staff) who had been in the same medical facilities at the same time or during the two hours after the case had visited. These people were contacted, counselled, and assessed for risk of infection. Immunoglobulin was recommended for three children under the age of 12 months, and three staff who cared for immunocompromised patients were placed on non-clinical care duties until their measles immunity was assessed by serology.

The SWSPHU provided the school with a letter advising all staff and students of possible exposure to measles. No further cases were reported. 


\section{QUARTERLY REPORT: AUSTRALIAN CHILDHOOD IMMUNISATION REGISTER}

Table 2 details the percentage of fully immunised children aged 12 months to less than 15 months in each Area Health Service, reported by all service providers.

These data refer to five different cohorts of children whose age has been calculated 90 days before data extraction.
The information contained in each of the reports has been extracted from the Australian Childhood Immunisation Register (ACIR) and may not reflect actual coverage due to under-reporting. Table 3 details the percentage of fully immunised children identified as Aboriginal or Torres Strait Islander in New South Wales for the same cohort, reported by all service providers.

\section{TABLE 2}

PERCENTAGE OF FULLY IMMUNISED CHILDREN AGED 12 MONTHS TO LESS THAN 15 MONTHS BY AREA HEALTH SERVICE

\begin{tabular}{|c|c|c|c|c|c|}
\hline Area Health Service & 30 June 02 & 30 Sept 02 & $31 \mathrm{Dec} 02$ & 30 March 03 & 30 June 03 \\
\hline Central Coast & 90 & 92 & 93 & 93 & 92 \\
\hline Central Sydney & 89 & 90 & 90 & 91 & 90 \\
\hline Hunter & 94 & 93 & 94 & 94 & 95 \\
\hline Illawarra & 89 & 94 & 92 & 92 & 93 \\
\hline Northern Sydney & 89 & 91 & 91 & 90 & 91 \\
\hline South Eastern Sydney & 89 & 92 & 91 & 90 & 91 \\
\hline South Western Sydney & 90 & 90 & 92 & 91 & 90 \\
\hline Wentworth & 90 & 91 & 90 & 93 & 91 \\
\hline Western Sydney & 90 & 91 & 92 & 92 & 90 \\
\hline Far West & 90 & 90 & 89 & 93 & 88 \\
\hline Greater Murray & 92 & 94 & 93 & 92 & 94 \\
\hline Macquarie & 93 & 91 & 92 & 92 & 94 \\
\hline Mid North Coast & 90 & 88 & 90 & 90 & 89 \\
\hline Mid Western & 91 & 91 & 94 & 94 & 93 \\
\hline New England & 92 & 91 & 93 & 92 & 92 \\
\hline Northern Rivers & 84 & 84 & 85 & 85 & 84 \\
\hline Southern & 90 & 91 & 91 & 89 & 91 \\
\hline NSW & 90 & 91 & 91 & 91 & 91 \\
\hline Australia & 90 & 91 & 92 & 91 & 91 \\
\hline
\end{tabular}

\section{TABLE 3}

PERCENTAGE OF FULLY IMMUNISED CHILDREN IDENTIFIED AS ABORIGINAL ANDTORRES STRAIT ISLANDER, AGED 12 MONTHS TO LESS THAN 15 MONTHS

\begin{tabular}{|lccccc|}
\hline & 30 June 02 & 30 Sept 02 & 31 Dec 02 & 31 March 03 & 30 June 03 \\
\hline NSW & 87 & 85 & 86 & 86 \\
Australia & 85 & 85 & 84 & 86 \\
\hline
\end{tabular}


FIGURE 2

\section{REPORTS OF SELECTED COMMUNICABLE DISEASES, NSW, JANUARY 1998 TO MAY 2003,} BY MONTH OF ONSET

Preliminary data: case counts in recent months may increase because of reporting delays. Laboratory-confirmed cases only, except for measles, meningococcal disease and pertussis $\mathrm{BFV}=$ Barmah Forest virus infections, RRV $=$ Ross River virus infections $\mathrm{LI}=$ Legionella longbeachae infections, $\mathrm{L} p=\mathrm{L}$. pneumophila infections Gp $C$ and $G p B=$ disease due to serogroup $C$ and serogroup B infection, other/unk $=$ other or unknown serogroups

\begin{tabular}{|rc|}
\hline \multicolumn{3}{|c|}{ NSW population } \\
Male & $50 \%$ \\
$<5$ & $7 \%$ \\
$5-24$ & $28 \%$ \\
$25-64$ & $52 \%$ \\
$65+$ & $13 \%$ \\
Rural $^{*}$ & $42 \%$ \\
\hline
\end{tabular}

\begin{tabular}{|rc|}
\hline \multicolumn{2}{|c|}{ Mar-May 03} \\
Male & $48 \%$ \\
$<5$ & $1 \%$ \\
$5-24$ & $6 \%$ \\
$25-64$ & $80 \%$ \\
$65+$ & $12 \%$ \\
Rural & $97 \%$ \\
\hline
\end{tabular}

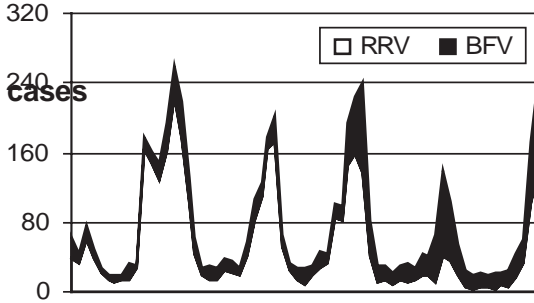

Arbovirus

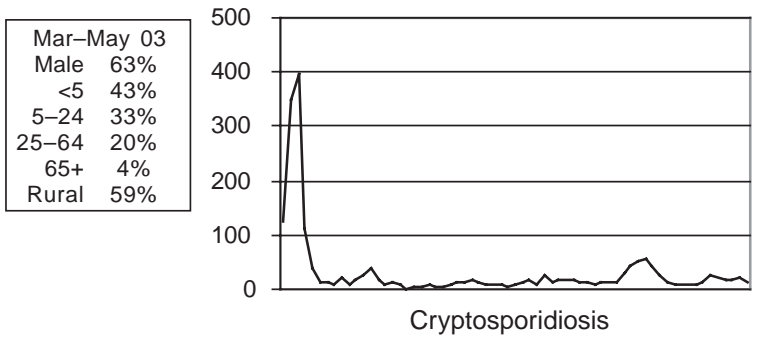

\begin{tabular}{|rc|}
\hline \multicolumn{2}{|c|}{ Mar-May 03} \\
Male & $87 \%$ \\
$<5$ & $<1 \%$ \\
$5-24$ & $22 \%$ \\
$25-64$ & $76 \%$ \\
$65+$ & $<1 \%$ \\
Rural & $16 \%$ \\
\hline
\end{tabular}

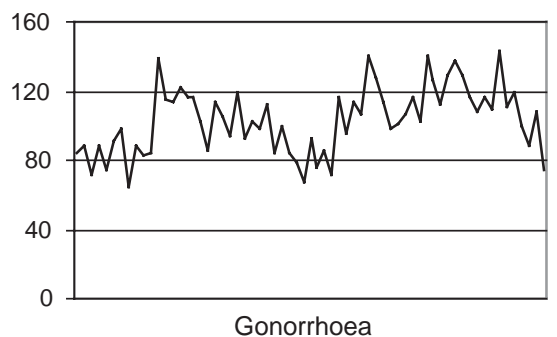

\begin{tabular}{|rc|}
\hline \multicolumn{2}{|c|}{ Mar-May 03 } \\
Male & $57 \%$ \\
$<5$ & $3 \%$ \\
$5-24$ & $43 \%$ \\
$25-64$ & $50 \%$ \\
$65+$ & $3 \%$ \\
Rural & $17 \%$ \\
\hline
\end{tabular}
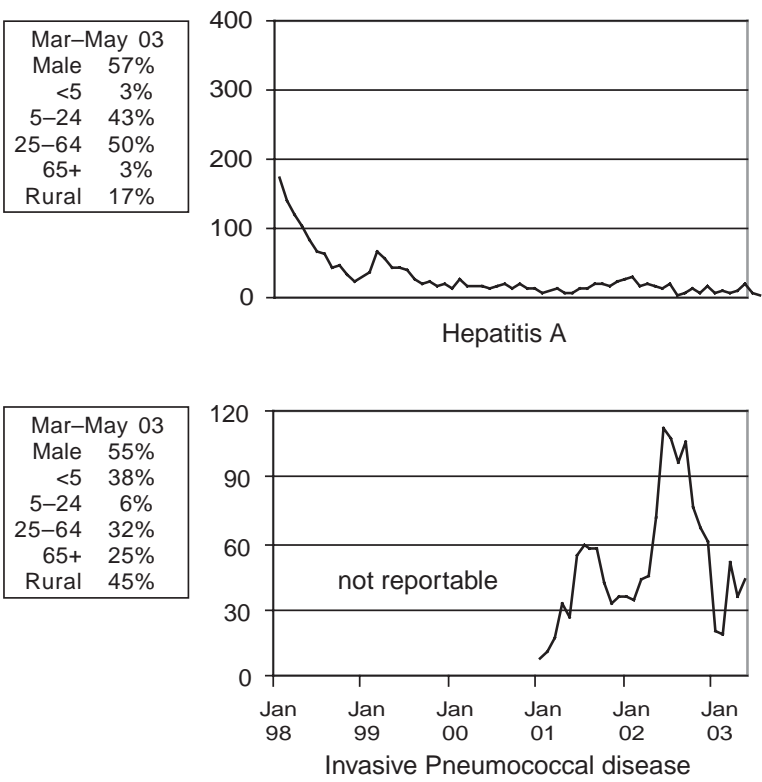

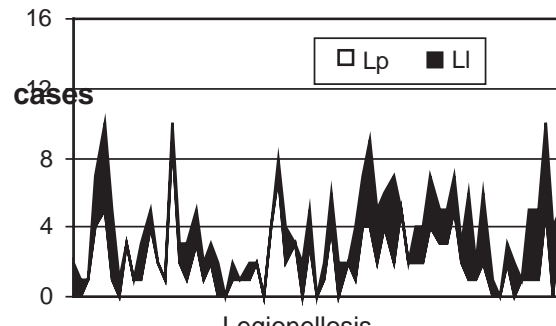

Legionellosis
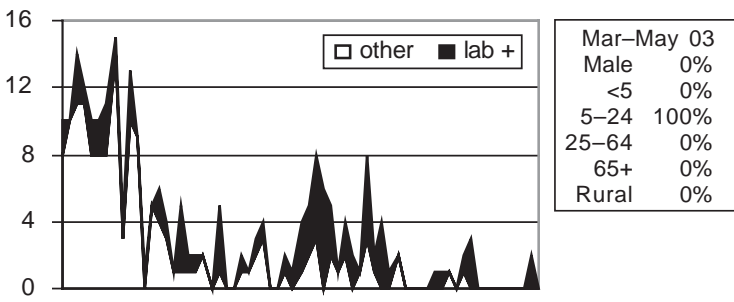

Measles

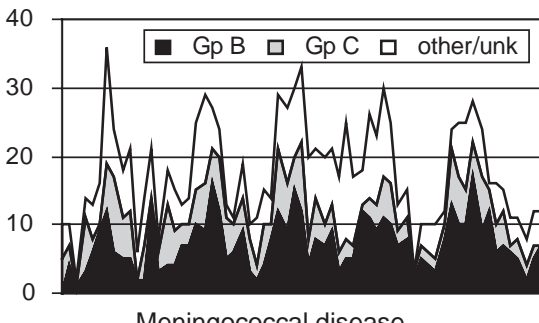

Mar-May 03 Male $56 \%$ $<5$
$5-24$
$34 \%$ 25-64 25\% $65+13 \%$ Rural $59 \%$
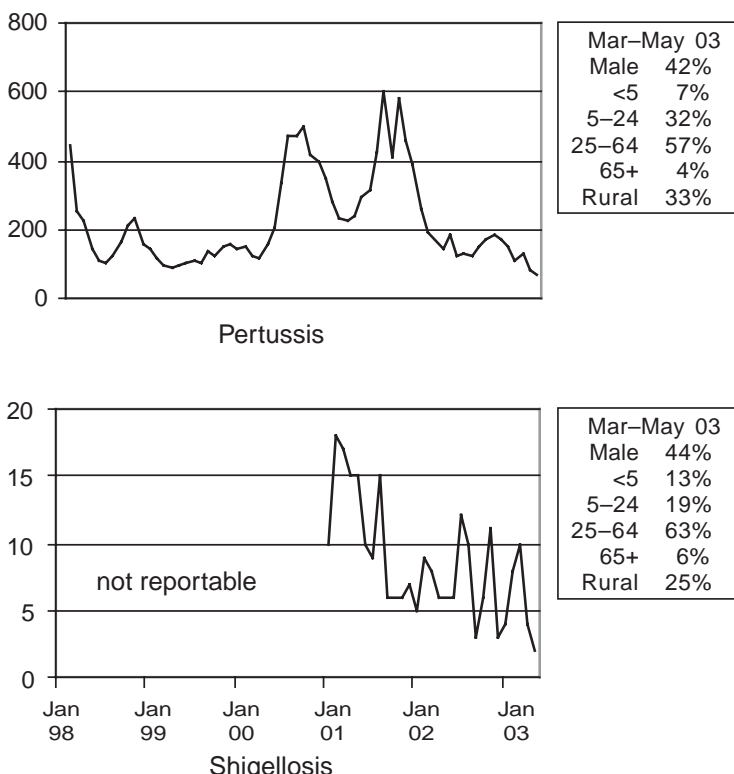


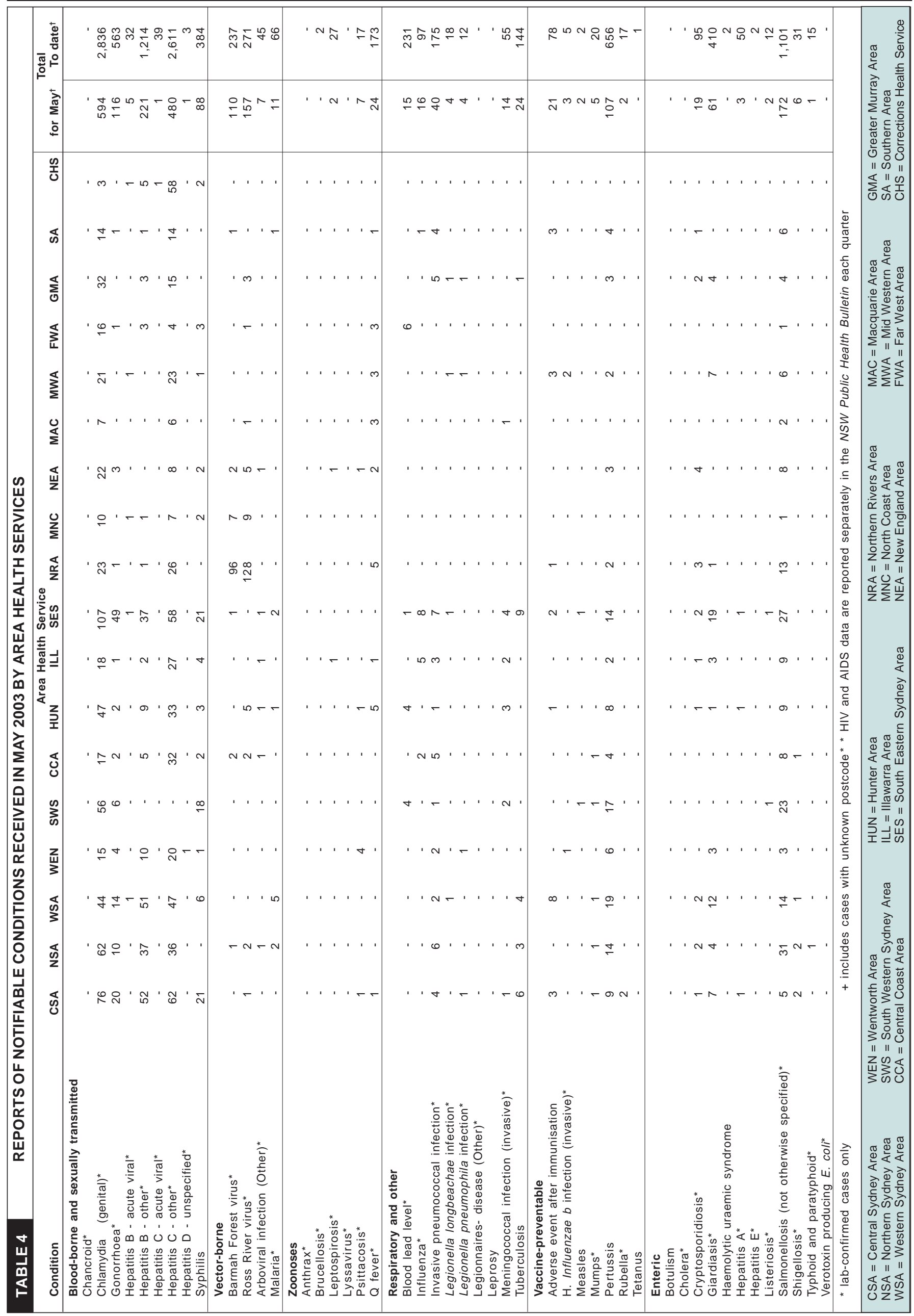

